impairments at the base-line interview. The sequence in which the defects are expressed in time may shed some light on the basic mechanisms that underly their co-occurrence.

For our report we have studied the crosssectional data (base-line interview). We found that a history of a prior impairment in the affective realm singled out a group of elderly subjects. Among these subjects GMS-AGECAT depression was not associated with MMSE scores below 26 . Among other elderly subjects these measurements were clearly associated, but the meaning of this relationship remains unclear.

The relationship may suggest that the MMSE impairment does, as a rule, not follow the onset of affective problems in time. Instead, the MMSEimpairment may accompany depressive disorders with a first onset in late life, occurring simultaneously. This conclusion is in line with the results of studies in which an increased rate of brain-scan abnormalities among elderly patients with a lateonset depression has been reported. The combination of late age, depression and mild impairment of MMSE-performance may indicate a specific and possibly organic syndrome that may be different from other cases of late life depression.

As Lester \& Blanchard point out, 'prodromal' Alzheimer's disease is only one of the possible explanations. Functional impairment, use of medication, gender, ethnicity or anxiety may also be involved, and exploration of the roles of these variables certainly seems worthwhile.

It would be interesting to study associations of the syndrome with such variables, but also with possible symptoms of depression other than anxiety, social stress-factors and medical diagnoses. Equally interesting may be the course of the syndrome. The course may be chronic or intermittent or a dementing illness may develop. The pathological anatomical abnormalities that may be associated with the syndrome also deserve some attention. These may be similar to those found among patients with Alzheimer's disease, but one would expect the localisation of abnormalities to be different at least.

Psychiatric Division of the

R. L. VAN OJEN

W. vaN TILBURG

Free University of Amsterdam

Valeriusclinic Amsterdam

\section{Keeping count for the community}

SIR: Wilkinson et al (1995) have provided a service to a catchment area with 14 stable schizophrenic patients living in it. The service consisted of one consultant psychiatrist, one senior registrar, 12 psychiatric nurses, one clinical psychologist, one senior occupational therapist, one senior social worker and two full time equivalent administrative assistants. This is in addition to primary care resources.

In the catchment area for which I am personally responsible I am aware of 180 people suffering from schizophrenia. Our team consists of myself, four-and-a-half-equivalent community psychiatric nurses, one untrained support worker and a halftime equivalent occupational therapist. Should similar resourcing be available to us there would be 12 consultant psychiatrists, 12 senior registrars, 144 psychiatric nurses, 12 clinical psychologists, 12 senior occupational therapists, 12 senior social workers and 24 full time equivalent administrative assistants. I am sure all 228 of us would do a jolly good job but how on earth would I learn all their names.

Wilkinson, G., Piccinelli, I., Falloon, I., et al (1995) An evaluation of community-based psychiatric care for people with treated long-term mental illness. British Journal of Psychiatry. 167, 26-37.

Greenwich District Hospital

M. BLACKWELL

Greenwich SE10 9HE

\section{Severe apathetic hyperthyroidism with normal} thyroid hormone levels

SIR: "Subclinical" hyperthyroidism (suppressed TSH secretion in the presence of normal thyroid hormone levels) is associated with an increased rate of physical and psychiatric complaints resembling those seen in overt hyperthyroidism (Scholte $e t$ al, 1992) and of other complications such as atrial fibrillation (Sawin et al, 1994). Severe apathy is well recognised as an uncommon complication of overt hyperthyroidism, but to our knowledge it has not previously been reported in a patient with normal circulating thyroid hormone levels.

Case report. A 62-year-old woman with a previous history of manic-depressive illness was admitted to hospital with a 5-month history of inactivity and progressive self-neglect. When she first became inactive the neuroleptic she was then receiving was discontinued but she deteriorated further and a presumptive diagnosis of depression was made. She did not comply with antidepressants and was admitted under the Mental Health Act in a dirty and neglected state with untreated infections of her eyes and urinary tract, and infestation with head lice. After admission she was profoundly apathetic, with 